\title{
One small step for mice, one giant leap for CWAS?
}

\author{
Mark A. Herman, ${ }^{1,2}$ Jonathan E. Campbell, ${ }^{1,2}$ and David A. D'Alessio ${ }^{1}$ \\ 'Duke Molecular Physiology Institute, Department of Medicine, Division of Endocrinology, and 'Department of Pharmacology and Cancer Biology, Duke University, Durham, North Carolina, USA.
}

\begin{abstract}
Genome-wide association studies (CWAS) have provided a wealth of information on potential disease-associated genes in the human population. In particular, several loci have been associated with type 2 diabetes (T2D). However, due to the complexity of the disease, it has been a challenge to unravel the exact effects of specific loci on T2D pathogenesis. In this issue of the $J C l$, Keller and colleagues developed a systems genetic approach to identify insulin secretion-associated genes in nondiabetic mice followed by tissue-level and functional phenotyping. Several of the loci identified were syntenic with human T2D-related loci, indicating that this approach may be feasible for discerning genetic variation in nondiabetic individuals that may lead to the development of T2D.
\end{abstract}

to describe human diabetes (3). Here they pursue a systems genetic approach to connect gene variants with insulin secretion in a mouse model that allowed for tissue-level and functional phenotyping of a dynamic process. These investigators took advantage of an outbred colony of mice (4) to perform a murine GWAS that used $\beta$ cell insulin secretion as the associated outcome. Nearly 500 male and female mice were genotyped at approximately 150,000 single nucleotide polymorphisms (SNPs), providing a high degree of genetic resolution. Insulin secretion was determined in single islets that were isolated from each mouse and incubated with a panel of secretagogues to generate a comprehensive response profile. None of the measures of ex vivo insulin secretion associated with quantitative trait loci (QTL) conferred large effects. This recapitulates the small impact of any single locus identified through GWAS in human populations and is consistent with the complex, polygenic nature of T2D. However, using genomewide scans for each secretagogue, Keller et al. were able to identify 60 QTL, of which five exceeded genome-wide significance. Insulin secretion QTL clustered at several hotspots that were associated with responses from multiple stimuli, as well as loci specific to single secretagogues.

Importantly, human loci syntenic to mouse loci associated with ex vivo insulin secretion were shown to be enriched for variants associated with $\mathrm{T} 2 \mathrm{D}$ in humans. This finding lends experimental credence to the previous inference that diabetes genes identified in GWAS relate to $\beta$ cell function. This is remarkable, since the genetically heterogeneous mice used in the study were uniformly nondiabetic. Moreover, mouse models do not develop islet pathology that mimics the features of human T2D. The overlap of genetic correlates of $\mathrm{T} 2 \mathrm{D}$ with those of murine $\beta$ cell function supports the application of this outbred mouse model to study human disease.

Once Keller and coworkers successfully demonstrated genetic correlates of $\beta$ cell function, they sought to identify the 
specific genes that mediate the observed physiologic effects. The authors pursued genes within causative loci whose expression correlated with both islet function and associated gene markers. Using this approach, they identified Ptpn18, a protein tyrosine phosphatase, as a factor that likely enhances insulin secretion. To validate the physiologic significance of this gene, Keller et al. created a mouse model expressing a catalytically inactive Ptpn18 enzyme and confirmed that reducing Ptpn18 activity reduces insulin secretion ex vivo. Similarly, they observed compatible findings with two other loss-of-function models, based on genes derived from QTL identified in the genome scan plus islet phenotype analysis. Increased expression of kinase Hunk or transcription factor $Z f p 148$ associated with reduced insulin secretion from isolated islets in outbred mice. The predicted increase in insulin secretion was validated in experiments using islets in static culture and perifusion experiments from $\mathrm{Hunk}-\mathrm{KO}$ and $Z f p 148$-KO mice. With these results, Keller et al. successfully closed the loop, demonstrating a connection between specific targets identified by genome scan and functional $\beta$ cell phenotypes. This set of observations supports the premise that genome-wide interrogation of functional phenotypes may produce insight into normal and pathologic physiology. Of note, two of the candidate genes validated in this analysis, Ptpn18 and Hunk, are involved in the HER2 signaling pathway (5). This pathway has not been reported to be involved in $\beta$ cell function, but has been implicated in the contribution to insulin resistance (6).

While Keller et al. address one of the problems that has stymied human GWAS, namely placing candidate genes in tissues that can affect key biologic processes, they have unmasked other hurdles that limit mechanistic insight from genetic screens. The in vivo experiments with loss-offunction mouse models to test selected candidates exemplify these limitations. The genome scan suggested that reducing Ptpn18 expression or activity should reduce insulin secretion, which was observed in islet studies. However, in vivo, the reduction in Ptpn18 activity had no obvious effect on metabolic physiology in chowfed mice and was associated with reduced weight gain and improved insulin sensitivity in high-fat fed mice. Thus, squaring the in vivo response of these animals to high-fat diet with the ex vivo insulin secretion results requires some unconventional hypotheses. Based on the conventional view of diabetes pathogenesis, caloric excess and weight gain on an obesogenic diet lead to insulin resistance with compensatory hyperinsulinemia. Hyperinsulinemia, through its enhancement of adipose lipid storage, may further exacerbate the development of obesity, and glucose intolerance develops if insulin secretion cannot compensate for insulin resistance. Therefore, reducing insulin secretion by inactivating Ptpn18 might have been expected to lead to glucose intolerance on a high-fat diet. However, the phenotype in mutants did not comport at all with this commonly held and well-supported model. One interpretation of these interesting results is that restraining insulin secretion mitigates weight gain in an obesogenic setting, a hypothesis that could be readily addressed with additional experiments. However, this result illustrates the complexity of deciphering the impact of even well-defined genetic manipulation using parameters of systemic physiology as the outcome. Extending human GWAS results to mechanisms of normal and disease physiology is almost entirely dependent on in vivo outcome measures, a limitation that does not have a ready solution.

\section{Remaining questions}

The difficulty in interpreting putative validation studies in preclinical models is also a function of choices in the design of experimental models and methods. For instance, associations between variants in the Ptpn18 locus and the insulin secretion phenotype were driven primarily by a haplotype from the wild-derived CAST mouse, in which increased Ptpn18 expression was associated with increased insulin secretion. Keller et al. opted to validate the function of Ptpn18 by generating a loss-of-function mutation in Ptpn 18 in C57BL/6 mice, a fairly standard approach. However, expression of Ptpn18 is typically low in C57BL/6 mice (7). There is an open question as to whether further reduction of Ptpn18 in a mouse that has constitutively reduced activity is an optimal test of this gene to affect insulin secretion. Would generating a model to increase $\beta$ cell Ptpn18 expression in a background with low expression be a better test of the results of the genome-wide scan? Would the effects of increasing as opposed to decreasing insulin secretion on systemic metabolism in response to an obesogenic challenge be reciprocal? These questions are both theoretical and empiric, but demonstrate the gaps in understanding that lurk beneath targets derived from genomic analysis.

A further limitation of interpreting the in vivo validation study of Ptpn18 is that the activity of the gene product was reduced throughout the many tissues that express it, and not specifically in $\beta$ cells. Perhaps the relative leanness in Ptpn18 loss-of-function mice is due to actions of Ptpn18 outside of $\beta$ cells? If so, the reduced insulin secretion in Ptpn18 mutant islets both in vivo and ex vivo could be secondary to leanness and not a direct result of changes in Ptpn18 activity within the islet. This points to a general concern with the assumption that the primary effect of a variant on a functional phenotype is cell specific and not an indirect response to its expression in other tissues. Because many genes, including Ptpn18, are expressed in multiple tissues, sorting disease mechanisms and relative strengths of effect from these sites is a substantial challenge.

The validation studies of Hunk and Zfp148 illustrate similar difficulties with the interpretation of physiology based on the outcomes of a genomic screen. Both of these genes were selected from the screen of outbred mice as likely inhibitors of insulin secretion. Indeed, knock out of these genes increased glucose-stimulated insulin secretion from isolated islets in both instances. It is worth noting that these ex vivo results were confirmed using perifusion of multiple islets from knockout and control lines and frequent sampling for insulin, a robust test of $\beta$ cell function. Despite the concordance of the ex vivo studies, oral glucose tolerance testing in knockout mice was dramatically different. Selective deletion of $Z f p 148 \beta$ cells caused substantial improvement in glucose tolerance, a predictable outcome of enhanced $\beta$ cell insulin secretion. The only modestly higher insulin concentrations in the knockout animals occurred at lower blood glucose, in keeping with enhanced secretory function. In contrast, mice with global Hunk deletion were glucose intolerant after high-fat feeding and had reduced insulin secretion in vivo. One explanation for these 
results is that $Z f p 148$ was selectively deleted in $\beta$ cells, whereas Hunk was not, such that the effect of $Z f p 148$ on insulin secretion is not sullied by effects of knockout in other tissues. While tissue-specific gene deletion has clear advantages for confirming a physiologic effect of a candidate in a specific tissue, it is not necessarily true that the physiologic effect of the candidate identified in human GWAS is conferred by its activity in that tissue.

\section{Concluding remarks}

Keller et al. conclude from their exciting results that defining the genetic architecture of insulin secretion among nondiabetic humans may lead to important insights into insulin secretion and T2D. However, even with robust phenotyping it seems likely that genomic targets identified in nondiabetic people will face the same limitations for validation that have restricted physiologic insights from GWAS in persons with T2D. We see the novel and refined method for target discovery as a major advance in this set of results, rather than a means to unravel human GWAS results. The com- bination of expansive genetic variation and tissue-specific phenotyping illuminated the involvement of at least one new and previously unknown signaling pathway (HER2) in insulin secretion. Certainly, this effort involving high-throughput screening required considerable technical expertise and led to the generation of a huge amount of data with a modest number of animal subjects relative to GWAS standards. It is reasonable to suppose that similar approaches can be taken to connect gene variants to the function of cell types and tissues beyond the $\beta$ cell for discovery in a range of physio-pathologic conditions. In fact, this approach is also amenable to translation. An obvious extension of the results of Keller and colleagues is to study signaling related to HER2, Ptpn18, Zfp148, and Hunk in human islets. This retrotranslational approach seems to have decided potential for expanding our understanding of the mechanisms regulating insulin secretion and diabetes.

Address correspondence to: David D'Alessio, 300 N. Duke Street, Duke Molecular
Physiology Institute, Division of Endocrinology, Duke University, Durham, North Carolina 27701, USA. Phone: 919.684.5778; Email:david.d'alessio@duke.edu.

1. Langenberg C, Lotta LA. Genomic insights into the causes of type 2 diabetes. Lancet. 2018;391(10138):2463-2474.

2. Dimas AS, et al. Impact of type 2 diabetes susceptibility variants on quantitative glycemic traits reveals mechanistic heterogeneity. Diabetes. 2014;63(6):2158-2171.

3. Keller MP, et al. Gene loci associated with insulin secretion in islets from nondiabetic mice. J Clin Invest. 2019;129(10):4419-4432.

4. Churchill GA, et al. The Diversity Outbred mouse population. Mamm Genome. 2012;23(9-10): 713-718.

5. Wang HM, et al. The catalytic region and PEST domain of PTPN18 distinctly regulate the HER2 phosphorylation and ubiquitination barcodes. Cell Res. 2014;24(9):1067-1090.

6. Memon AA, et al. Circulating human epidermal growth factor receptor 2 (HER2) is associated with hyperglycaemia and insulin resistance. J Diabetes. 2015;7(3):369-377.

7. DiGruccio MR, et al. Comprehensive alpha, beta and delta cell transcriptomes reveal that ghrelin selectively activates delta cells and promotes somatostatin release from pancreatic islets. $\mathrm{Mol}$ Metab. 2016;5(7):449-458. 